\title{
Politique
}

Politique

Espace urbain et différenciation sociale au Saguenay :

l'agglomération de Chicoutimi-Jonquière du Groupe ECOBES, Jonquière, 1989, 137 p.

À la périphérie de la périphérie : l'espace rural et le concept de fragilité en Abitibi de Hervé Gumuchian, Notes et documents, $n^{0}$ 90-01, Département de géographie, université de Montréal, 1990, 66 p.

\section{Maurice Couture}

Numéro 20, automne 1991

URI : https://id.erudit.org/iderudit/040707ar

DOI : https://doi.org/10.7202/040707ar

Aller au sommaire du numéro

Éditeur(s)

Société québécoise de science politique

ISSN

0711-608X (imprimé)

1918-6584 (numérique)

Découvrir la revue

Citer ce compte rendu

Couture, M. (1991). Compte rendu de [Espace urbain et différenciation sociale au Saguenay : l'agglomération de Chicoutimi-Jonquière du Groupe ECOBES, Jonquière, 1989, 137 p. / À la périphérie de la périphérie : l'espace rural et le concept de fragilité en Abitibi de Hervé Gumuchian, Notes et documents,

$\mathrm{n}^{0}$ 90-01, Département de géographie, université de Montréal, 1990, 66 p.]

Politique, (20), 174-176. https://doi.org/10.7202/040707ar d'utilisation que vous pouvez consulter en ligne. 
Espace urbain et différenciation sociale au Saguenay : l'agglomération de Chicoutimi-Jonquière du Groupe ECOBES, Jonquière, 1989, 137 p.

\section{À la périphérie de la périphérie :}

l'espace rural et le concept de fragilité en Abitibi de Hervé Gumuchian, Notes et documents, $n^{\circ}$ 90-01, Département de géographie, université de Montréal, 1990, 66 p.

Les études régionales s'étaient essoufflées, celles des sociologues et des politologues. Voilà qu'en moins d'une année des géographes (même s'il n'y a pas que des géographes dans l'équipe ECOBES, la méthode est inspirée de la discipline), avec leur ambition d'être au carrefour des sciences sociales, nous offrent ces rapports de recherche sur deux régions périphériques du Québec. Quoi de nouveau? Quoi d'utile? Fallait-il tant d'efforts pour se faire dire que les régions périphériques sont fragiles et que les pauvres sont davantage malades et les municipalités sans ressources plus précaires? Ce n'est évidemment pas dans la réponse brute que s'évalue l'intérêt des deux rapports. Leur pertinence déborde les résultats exposés.

Dans les deux cas, on retrouve cette préoccupation des chercheurs pour les problèmes quotidiens des citoyens qui vivent dans ces espaces spécifiques. Dans les deux cas, on ne retient qu'une partie de la région officielle. Dans les deux 
cas, la région est perçue et vécue comme un «isolat» ou une "ile" par rapport à l'espace québécois.

La recherche du groupe ECOBES-Saguenay se limite à la conurbation Chicoutimi-Jonquière. Elle a une orientation pratique : tracer le profil sociosanitaire de la région du Saguenay. Le contenu est plutốt classique : évolution démographique, distribution résidentielle selon les caractéristiques socio-économiques. La particularité et l'avantage, pour les auteurs de l'étude, tiennent a l'échelle du traitement des données, soit le niveau microgéographique. En effet, les auteurs utilisent la plus petite unité géostatique disponible du recensement canadien, le secteur de dénombrement'. S'inscrivant dans la foulée de l'écologie factorielle, ils situent ce rapport dans leur démarche de connaissance de l'état de santé d'une population comme étant "l'aboutissement de travaux visant à élaborer un modèle d'analyse pour comprendre la logique résidentielle et pour repérer les disparités sociosanitaires existantes" (Avant-propos). En fait, l'objectif est plus ambitieux puisqu'ils cherchent à «mettre en évidence la capacité prédictive d'une telle approche en matière de santé»" (p. 104). Cette étude sur le Saguenay comporte cet intérêt non négligeable de compléter, voire confronter, d'autres études utilisant le même cadre spatial, notamment celle de L. M. Bouchard (1973) ou encore celles du même groupe réalisées depuis 1984. C'est ici qu'on peut trouver l'explication de la référence au recensement de 1981 - au lieu de 1986 - pour la majorité des tableaux, des figures et des cartes (45) dans ce document de 137 pages. Une mise à jour eût été souhaitable vu l'intention de suivre de près l'évolution de la situation.

Si à partir des données de recensement on veut mieux appréhender la logique spatiale des citoyens pour l'offre de services sociosanitaires, la démarche est opposée pour l'étude sur l'Abitibi. La santé est encore au cœur de la préoccupation du chercheur, mais il s'agit de celle des municipalités qui, par ricochet, a un effet sur celle de ses citoyens. Démarche

1. A cet égard, nous regrettons que la majorité des cartes, figures et tableaux soient ceux de 1981 au lieu de 1986. 
inverse dans ce sens où l'auteur privilégie tout autant le regard intérieur que celui de l'extérieur pour appréhender la pluralité des espaces abitibiens, et les divers discours sur cet espace.

Dépassant le modèle centre-périphérique avec la notion classique induite qu'est la marginalité, l'auteur opte pour le concept de fragilité «du milieu humain*, kdes rapports que les groupes entretiennent avec l'espace* (p. 6). Ce choix l'oblige a construire un indice de fragilité à partir de neuf indicateurs additionnés pour les quelque 52 municipalités des 4 municipalités régionales de comté de l'Abitibi. L'opération propose une stratification en trois classes de municipalités : très fragiles, fragiles et autres. Une restitution cartographique de l'application de l'indice est d'ailleurs proposée pour les lecteurs trop pressés de comprendre la démarche et les causes de la fragilité, et pour les décideurs en mal de solutions hâtives (quatre figures p. $26-32$ ).

L'enquête par questionnaire auprès d'un échantillon de la population des dix-sept municipalités classées ufragiles" met en exergue la pertinence et l'urgence de prendre en compte "le regard de l'intérieur" (p. 4) et illustre ainsi comment, *entre espoir et désespérance, se construisent de nouvelles territorialités» (p. 3) et où apparaissent de nouvelles formules de mise en valeur et de gestion de l'espace.

Ces deux rapports de recherche constituent un apport significatif aux études régionales. La pertinence devient enfin un critère d'enquête et de croisement de données. Dans le premier cas, en faisant voir à la loupe une réalité complexe, et, dans le second, en démontrant que périphérie n'est plus synonyme de mort lente.

Maurice Couture Université du Québec a Montréal 